\title{
Venous Malformations in Children: When to Operate?
}

\author{
Mohamed Ramadan Abdallah, $M D^{1}$; Karam Allam, $M D^{2}$; \\ Omar Abdelrahim, $M D^{3}$; Tarek Fetohi, MD $^{4}$.
}

\author{
1) Pediatric Surgery Unit, General Surgery Department, Sohag University. \\ 2) Cleft/Craniofacial Unit, Plastic Surgery Department, Sohag University. \\ 3) General Surgery Department, Sohag University. \\ 4) Maxillofacial unit, General Surgery Department, Sohag University.
}

Background: Congenital venous malformations in children are common and their management can be challenging. Injection sclerotherapy and surgery are the main treatment modalities.

Patients and methods: The charts of 10 children with venous malformations who were treated by surgical excision of their lesions were reviewed.

Results: There were 6 males and 4 females with a mean age of 3.6 years. Lesions were located in the neck $(n=3)$, upper extremity $(n=3)$, trunk $(n=2)$ and lower extremity $(n=2)$. The main presentation was a growing mass lesion. Pain was reported in one patient and a sense of heaviness was reported in another patient. All lesions were of the discrete spongy type and their mean size was $5.8 \mathrm{~cm}$. Clinical examination and MRI were used for diagnosis. Complete surgical excision was achieved in all cases with a mean operative time of 80 minutes. The diagnosis of venous malformations was confirmed postoperatively by histopathologic examination. There was no recurrences during a mean follow up period of 2.3 months. All patients had satisfactory functional and cosmetic outcomes apart from two patients with wound related complications.

Conclusion: Surgical excision of venous malformations can be safe provided certain selection criteria are employed.

Key words: Vascular anomalies, vascular malformations, venous malformations.

\section{Background:}

Venous malformations (VMs) are slowflow vascular malformations, caused by abnormalities in the development of the veins. The malformation varies from simple varicosities and ectasias or discrete spongy masses, to a diffuse network of complex channels that permeate an organ or tissue. The main locations are the head and neck (40\%), trunk (20\%), and extremities (40\%). ${ }^{1}$

Sclerotherapy and surgery are the mainstay of treatment of venous malformation. We report our experience with 10 children with venous malformation who were treated by surgical excision of their lesions at Sohag university hospitals, over a period of 21 months [between January 2013 and September 2014]. The main objective of this study is to define the criteria of the venous malformation that can predict successful surgical excision.

\section{Patients and methods:}

The data of 10 children with venous malformations were retrospectively reviewed. Data included patients' gender, age at the time of surgery, location of the lesion and its size, the main presenting complaint, imaging studies used for diagnosis and/or preoperative mapping, the main reason for opting to surgery as the modality of treatment, the operative time, and the postsurgical cosmetic and functional outcomes.

\section{Results:}

Ten patients with venous malformations 
were treated surgically at Sohag University Hospitals over a period of 21 months [between January 2013 and September 2014]. There were 6 males $(60 \%)$ and 4 females $(40 \%)$. The patients' ages at the time of operation ranged from 2.1 to 6.2 years (mean 3.6 years). The lesion was located in the neck in 3 patients (30\%), upper extremity in 3 patients (30\%), trunk in 2 patients $(20 \%)$ and the lower extremity in 2 patients $(20 \%)$. A mass that was growing with the growth of the child was the presenting feature in all 10 patients $(100 \%)$. Episodic pain was reported in only one patient $(10 \%)$ with upper extremity venous malformation and was related to phlebolith. A sense of heaviness was reported in one patient (10\%) with lower extremity venous malformation.

All lesions presented as discrete masses that tended to have a circular or semicircular outline. Their sizes ranged from 3.4 to $8.2 \mathrm{~cm}$ (mean $5.8 \mathrm{~cm}$ ) at their maximum dimensions.

The diagnosis was made on a clinical ground supplemented by MRI in all 10 patients. MRI was used to confirm the diagnosis and delineate the relations of the lesion with the surrounding structures. None of the 10 patients had been subjected to any other treatment modality. In all 10 patients, surgery as the treatment modality was chosen based on two factors: the discrete nature of the lesion, and the MRI findings showing a clear plane between the lesion and surrounding structures. Complete excision of the lesion was achieved in all cases and the operative time ranged from 45 minutes to 150 minutes (mean 80 minutes). Wound closure under tension was faced in 2 patients $(20 \%)$, both had their lesions located in the trunk, their sizes were $>5 \mathrm{~cm}$ and both lesions had significant involvement of the skin that could not be spared. Wound disruption occurred in one of them within one week of the operation and required secondary closure. Scar spread after 6 months of the operation occurred in the other patient and was significant enough to require scar revision. Histopathologic examination of the operative specimens showed dilated venous channels with abnormal distribution of the smooth muscles in their walls, confirming the diagnosis of venous malformation in all patients. The follow up period ranged from 1 month to 8 months (mean 2.3 months). Apart from the 2 patients who had problems related to wound closure under tension, all patients had satisfactory functional and cosmetic outcomes. There was no recurrences during the follow-up period.

\section{Discussion:}

Vascular anomalies include vascular tumors (i.e. hemangiomas) and vascular malformations. The two categories are different regarding their appearance, growth pattern plus imaging, and histopathological characteristics. Vascular malformations include capillary malformations, venous malformations, lymphatic malformations, arteriovenous malformations, and their combinations. $^{2,3}$

Congenital venous malformations are common vascular malformations and are thought to be due to a localized developmental defect of the vasculature characterized by enlarged venous channels surrounded by sparsely distributed vascular smooth muscle. ${ }^{4,5}$

Venous malformations can be found in any part of the body and the lesion can be superficial, limited to the dermis and subcutaneous tissues or deeper and can even infiltrate muscle or bone. The malformation varies from simple varicosities and ectasias or discrete spongy masses, to a diffuse network of complex channels that permeate an organ or tissue. They can have anatomic, pathological and hemodynamic effects on the surrounding tissues and on the whole body. ${ }^{6}$ All 10 lesions selected for surgical excision in our study were of the discrete spongy type.

Apart from the mass lesion, the most common symptom is pain which can range form heaviness of the affected region to acute episodic pain due to thrombosis and calcification. ${ }^{7}$ Out of 10 patients included in this study, only one patient $(10 \%)$ had acute pain from thrombosis and another one $(10 \%)$ experienced a sense of heaviness in the lower extremity related to the lesion. This can be 

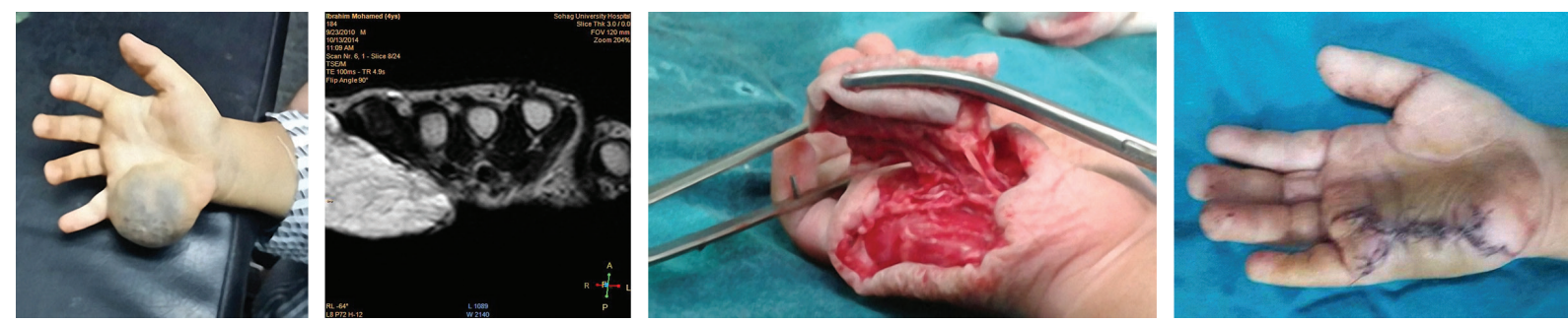

Figure (1): Clinical, radiographic and operative photographs of a patient with a hand venous malformation.
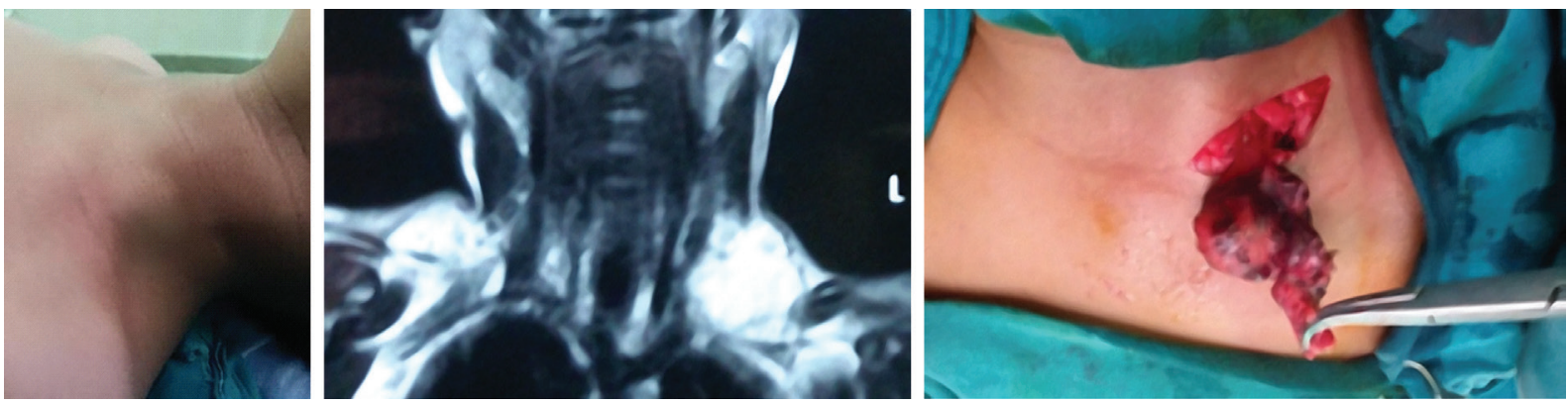

Figure (3): Clinical, radiographic and operative photographs of a patient with a neck venous malformation.

explained by the fact that all patients included in this study had localized or discrete lesions which tend to be painless, unlike the diffuse extensive forms of venous malformations that are often accompanied by venous pooling with its subsequent complications of heaviness and thrombosis.

Venous malformations

grow proportionately with the child and tend to expand slowly with time. ${ }^{6}$ The lesion may also enlarge and become symptomatic with trauma, puberty or during pregnancy. ${ }^{8}$ Diffuse venous malformations progress more often than localized lesions. In addition, lesions affecting the trunk and extremity are more likely to progress than head/neck malformations. This may be explained by the fact that VMs in dependent locations are prone to increased intralesional venous pressure from gravity, which likely contributes to venous pooling, distention, and stasis. ${ }^{9}$

Appropriate diagnosis and assessment of VMs can be made based on clinical presentation and a proper combination of basic non-invasive studies. Basic diagnostic tools are Duplex sonography followed by MRI. ${ }^{10}$ In our study, we depended on both clinical evaluation and MRI for diagnosis. We favored MRI over Duplex sonography because MRI is more informative. In addition to confirming the diagnosis of venous malformation, it provides significant information about the extent of the mass and its relation with the surrounding structures. Lesions are hyperintense with T2 sequences. Identifiable spaces separated by septations can be seen. The vascular spaces of venous malformations enhance with contrast, whereas lymphatic malformation does not; the exception to this rule is a lymphatic malformation with intralesional bleeding. Phleboliths are often seen as signal voids on all sequences. ${ }^{6}$

Although treatment of venous malformations is reserved for symptomatic patients, earlier therapy is now considered for asymptomatic lesions, especially if the venous malformation is in a difficult anatomic area (e.g., face, hand), because of the likelihood of progression making treatment more challenging. ${ }^{9}$ This has been the case in 8 out of 10 patients in our study who were completely asymptomatic apart from the presence of the mass. The age at operation ranged from 2.1 to 6.2 years (mean 3.6 years) and the size of the lesions ranged from 3.4 to $8.2 \mathrm{~cm}$ (mean $5.8 \mathrm{~cm}$ ). Having in mind that venous malformations are not static and that 
they have the potential to expand and become symptomatic over time, we operated at an early age and in asymptomatic patients.

Sclerotherapy is often considered the first-line treatment. Common sclerosants for venous malformations include dehydrated ethanol and detergent sclerosants such as sodium tetradecyl sulfate (STS), polidocanol, sodium morrhuate, and ethanolamine. Sclerosing agents cause direct endothelial damage, thrombosis, and scarring. Possible local complications are blistering, fullthickness cutaneous necrosis, and nerve injury. Systemic complications include hemolysis, sudden pulmonary hypertension, and cardiac and renal toxicities. ${ }^{11,12,13,14}$ Unfortunately, many VMs recanalize and expand after treatment, thus requiring additional procedures. ${ }^{6}$ None of our patients had been subjected to sclerotherapy before surgical excision.

When a lesion is localized, and does not involve vital structures, surgical excision alone can result in excellent outcomes with minimal risk of recurrence. Surgical resection of venous malformations may also be useful when sclerotherapy cannot be safely performed due to the location of the lesion or when a lesion is too large for effective sclerotherapy. ${ }^{15}$ In this series, lesions selected for surgical excision were of the discrete type of venous malformation based on the clinical evaluation and the MRI findings. Lesions were circumscribed and relatively easily accessible. We found that these discrete forms of venous malformations often preserve a good plane between them and the surrounding structures and often contain a considerable amount of fibrofatty tissue both around and within the substance of the mass which facilitates separation from the surroundings. The use of bipolar cautery for dissection, and staying as close to the mass as possible are of great importance for complete and safe surgical excision of these lesions.

\section{Conclusion:}

Many venous malformations in children are amenable to safe and complete surgical excision. The two important factors that can predict favorable postsurgical outcomes are the type of the lesion being of the discrete spongy type and the MRI findings of a clear plane between the lesion and structures surrounding it.

\section{Reference:}

1- Kumar S, Kumar V, Kumar S, et al: Management strategy for facial venous malformations. Natl J Maxillofac Surg 2014; 5(1): 93-96.

2- Mulliken JB, Glowacki J: Hemangiomas and vascular malformations in infants and children: A classification based on endothelial characteristics. Plast Reconstr Surg 1982; 69: 412-422.

3- Enjolras O, Mulliken JB: Vascular tumors and vascular malformations. Adv Dermatol 1997; 13: 375-423.

4- Brouillard P, Vikkula M: Genetic causes of vascular malformations. Hum Mol Genet 2007; 16 (2): 140-149.

5- Limaye N, Wouters V, Uebelhoer M, et al: Somatic mutations in angiopoietin receptor gene TEK cause solitary and multiple sporadic venous malformations. Nat Genet 2009; 41: 118-124.

6- Kulungowski AM, Fishman SJ: Vascular Anomalies. In: Pediatric Surgery. Coran AG, Adzick NS, Krummel TM, et al (Editors); USA: Elsevier Sciby (Publisher); $7^{\text {th }}$ edn. 2012; p.1613-1630.

7- Mazoyer E, Enjolras O, Laurian C, et al: Coagulation abnormalities associated with extensive venous malformation of the limbs: Differentiation from Kassabach-Merritt syndrome. Clin Lab Haematol 2002; 24: 243-251.

8- Duyka LJ, Fan CY, Coviello-Malle JM, et al: Progesterone receptors identified in vascular malformations of the head and neck. Otolaryngol Head Neck Surg 2009; 141: 491-495.

9- Hassanein AH, Mulliken JB, Fishman SJ, et al: Venous malformation: Risk of progression during childhood and adolescence. Ann Plast Surg 2012; 68(2): 198-201.

10- Lee BB, Antignani PL, Baraldini V, et al: ISVI-IUA consensus document - diagnostic guidelines on vascular anomalies: Vascular malformations and hemangiomas. Int Angiol 2014. [Epub ahead of print]

11- Burrows PE, Mason KP: Percutaneous treatment of low flow vascular malformations. 
J Vasc Interv Radiol 2004; 15: 431-445.

12- Legiehn GM, Heran MK: A step-by-step practical approach to imaging diagnosis and interventional radiologic therapy in vascular malformations. Semin Intervent Radiol 2010; 27: 209-231.

13- Dubois J, Alison M: Vascular anomalies: What a radiologist needs to know. Pediatr
Radiol 2010; 40: 895-905.

14- Behr GG, Johnson CM: Vascular anomalies: Hemangiomas and beyond-part 2: Slowflow lesions. Am J Roentgenol 2013; 200 : 423-436.

15- Dasgupta R: Surgical management of vascular anomalies. Curr Otorhinolaryngol Rep 2014; (2): 285-291. 


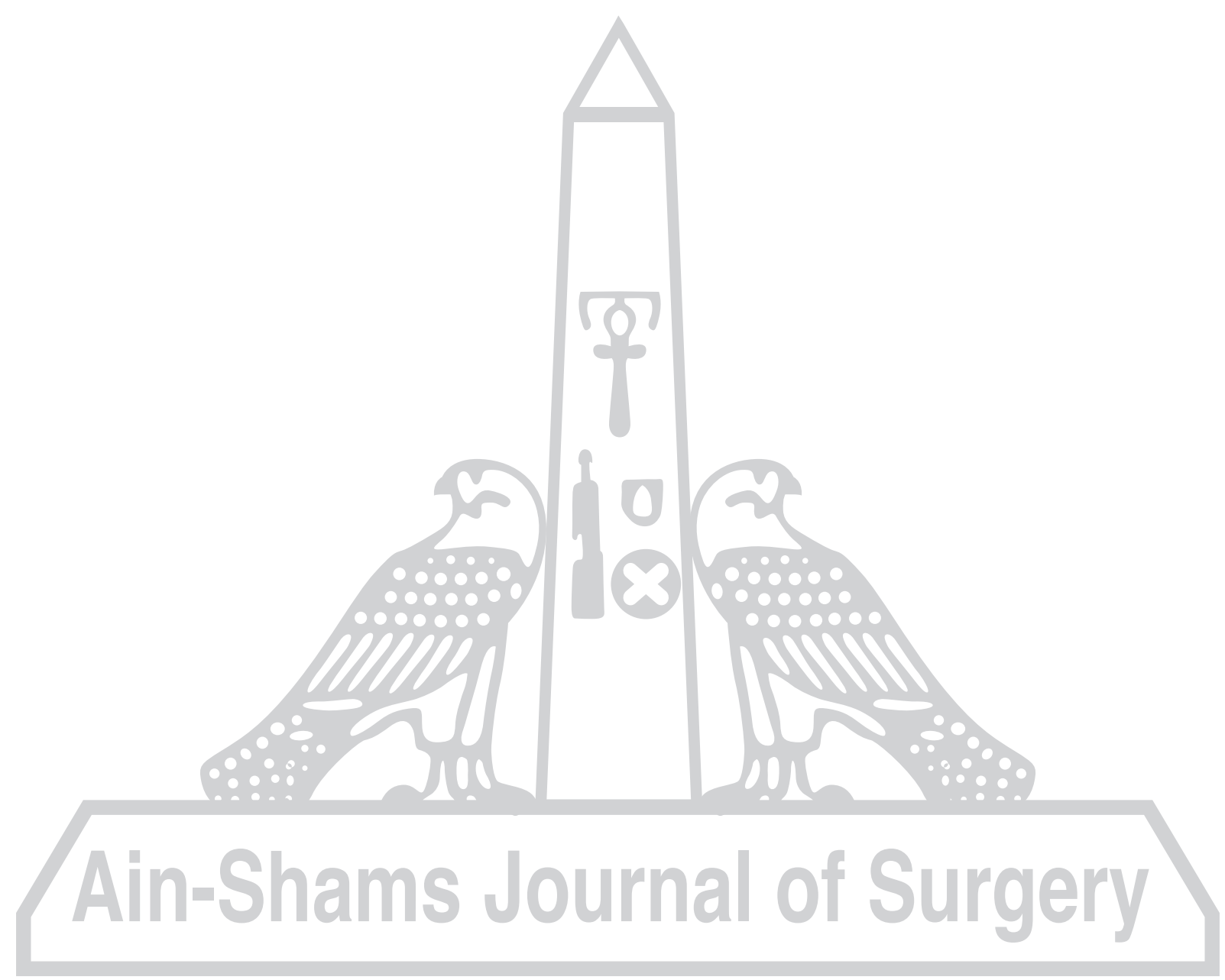

